\title{
Chancen und Herausforderungen der Rückversicherung in der gesetzlichen Krankenversicherung
}

\author{
Christoph Schwarzbach • Simone Krummaker • \\ J.-Matthias Graf v. d. Schulenburg
}

Online publiziert: 3. Februar 2012

(C) Springer-Verlag 2012

Zusammenfassung Bis zum 1. Januar 2009 wurden in der Gesetzlichen Krankenversicherung (GKV) aufwändige Leistungsfälle in einem gemeinsamen Risikopool der Krankenkassen quotal ausgeglichen. Dieser wurde durch den morbiditätsorientierten Risikostrukturausgleich (Morbi-RSA) abgelöst, welcher prospektiv ausgestaltet ist und die durchschnittlichen Folgekosten einer Behandlung ausgleicht. Seither sind im Bereich der GKV nur einzelne Rückversicherungsverträge abgeschlossen worden, die sich zudem meist auf deren Randbereiche beschränken. In diesem Artikel werden zunächst verschiedene Risikokonstellationen ermittelt, anhand derer die Natur der Krankheitsrisiken in der GKV analysiert und ihre Versicherbarkeit überprüft werden. Auf den Ergebnissen aufbauend werden die Potentiale von Rückversicherungslösungen auch in anderen Bereichen des Gesundheitsmarktes (z.B. Ärztenetze, Integrierte Versorgung) diskutiert.

Abstract In 2009 a so-called morbidity orientated risk structure equalization scheme was installed for the German statutory health insurance in order to minimize structural differences between different providers with respect to revenue and expenditures. Even with this mechanism some risks to the individual health insurance providers remain. Reinsurance could be a way to mitigate these risks, but so far

C. Schwarzbach $(\varangle) \cdot$ S. Krummaker · J.-M. Graf v. d. Schulenburg

Kompetenzzentrum Versicherungswissenschaften GmbH, Königsworther Platz 1, 30167 Hannover, Deutschland

e-mail: cms@ivbl.uni-hannover.de

J.-M. Graf v. d. Schulenburg

Institut für Versicherungsbetriebslehre, Gottfried Wilhelm Leibniz Universität Hannover,

Königsworther Platz 1, 30167 Hannover, Deutschland

S. Krummaker

University of East Anglia London / Norwich Business School, 102 Middlesex Street, London E1

7EZ, England 
only very few contracts have been signed. Moreover the existing reinsurance contracts only focus on the periphery of the statutory health insurance system such as travel health insurance. In this article we therefore analyse existing risks for individual health insurance providers and evaluate their (re-)insurability. Hereafter the potential for reinsurance solutions in the German statutory health insurance itself as well as in newer forms of healthcare provision (e.g. integrated health care and managed care) is discussed. We find that reinsurance may be a reasonable solution for many of the risks in the statutory health insurance scheme. But as research in this area is very young further analysis of the nature of risks is necessary.

\section{Einleitung}

Bis zum 1. Januar 2009 existierte in der Gesetzlichen Krankenversicherung (GKV) ein gemeinsamer Risikopool der Krankenkassen für aufwändige Leistungsfälle, in welchem überdurchschnittlich hohe Krankheitskosten einzelner Versicherter (für die stationäre Versorgung, Arznei- und Verbandsmittelversorgung, nichtärztliche Leistungen der ambulanten Dialyse sowie Kranken- und Sterbegeld) teilweise ausgeglichen wurden. In den Jahren 2002 und 2003 betrug der Schwellenwert 20.450 Euro pro Jahr und wurde in den Folgejahren angepasst. Ausgaben über diesem Betrag wurden zu 60 Prozent aus dem Risikopool ausgeglichen. ${ }^{1}$ Die Modalitäten des Risikopools entsprachen dabei für den über den Selbstbehalt hinausgehenden Betrag einer klassischen Quotenrückversicherung, die über einen Zwangspool organisiert wurde. ${ }^{2}$ Weil der Morbi-RSA theoretisch für einen Ausgleich zwischen guten und schlechten Risikostrukturen der Krankenkassen sorgt, wurde der Risikopool im Jahr 2009 abgeschafft. $^{3}$

Seither und vor allem seit einige gesetzliche Krankenversicherer in finanzielle Schwierigkeiten geraten sind, wird immer wieder von einer zunehmenden Relevanz und einem hohen Geschäftspotential von Rückversicherung im Bereich der GKV gesprochen. ${ }^{4}$ Allerdings finden sich in der praktischen Anwendung bisher nur sehr wenige Rückversicherungslösungen, welche in der Regel gut abgrenzbare Bereiche der Krankenversicherung, wie z.B. die Auslandsreisekrankenversicherung, abdecken. Daher gibt es zur Ausgestaltung solcher Risikoübernahmen in Deutschland nur wenige Erkenntnisse. Auch in einigen ausländischen Krankenversicherungsmärkten werden Rückversicherungsinstrumente eingesetzt. ${ }^{5}$ Rückversicherungen sind weltweit in der Lage, komplexe und auch große Risiken zu decken, zu transferieren und den

\footnotetext{
${ }^{1}$ Vgl. § 269 SGB V.

${ }^{2}$ Vgl. dazu auch Weber, A. (1989) und Weber, A. (1991), der bereits ein entsprechendes Rückversicherungskonstrukt für die GKV vorschlägt.

${ }^{3}$ Eine ähnliche Konstruktion ist aber auf freiwilliger Basis immer noch im Rahmen der jeweiligen Verbände der Krankenkassen möglich, vgl. § 265 SGB V. Ein Beispiel findet sich in BKK-Landesverband Nordwest (Hrsg.) (2011), Anlage „Ausgleichsordnung“.

${ }^{4}$ Vgl. Schlingensiepen, I. (2010).

${ }^{5}$ Bspw. ist die Munich Health in Abu Dhabi für Erst- und Rückversicherung eines PKV-ähnlichen Produktes verantwortlich. Vgl. Strassl, W. (2010), S. 747.
} 
erforderlichen Kapitalbedarf zur Verfügung zu stellen. Daher stellt sich die Frage, warum Rückversicherung als Instrumentarium der Risikoübernahme im Bereich der GKV eine so geringe Verbreitung aufweist.

Zur Annäherung an diese Frage, werden im Folgenden die Risiken im Standardangebot einer Krankenkasse analysiert und daraus mögliche Insolvenzszenarien generiert. Anschließend werden Kriterien der Versicherbarkeit von Risiken vorgestellt und diskutiert. Diese bilden die Basis der weiteren Analyse, indem die im zweiten Kapitel generierten Szenarien mit Hilfe der Versicherbarkeitskriterien überprüft werden. Die Arbeit schließt mit vorläufigen Ergebnissen zu Angebot und Nachfrage nach Rückversicherung im Bereich der GKV.

\section{Die Wirkung des Risikostrukturausgleichs auf Risiken der Krankenkassen}

\subsection{Charakteristika des Risikostrukturausgleichs}

Basierend auf dem Gesundheitsstrukturgesetz (GSG) aus dem Jahr 1992 sind die Versicherten der GKV seit dem Jahr 1996 nicht mehr den einzelnen Krankenkassen weitgehend fest zugeordnet, sondern können sich frei zwischen diesen entscheiden. Um eine Benachteiligung einzelner Kassen durch gewachsene Mitgliederstrukturen und eine zukünftige aktive Risikoselektion zu Gunsten tendenziell gesunder, junger und gut verdienender Versicherten zu vermeiden, wurde der Risikostrukturausgleich in einzelnen Schritten ab 1994 eingeführt. Dieser wird durch das Bundesversicherungsamt (BVA) gesteuert. Anfänglich erfolgte ein Ausgleich zwischen den Krankenkassen nach den Kriterien Einkommen, Zahl der beitragsfrei mitversicherten Familienangehörigen, Alter, Geschlecht, Berufs- und Erwerbsunfähigkeit sowie Krankengeldanspruch. Aber bereits 2001 wurde die Einführung eines morbiditätsorientierten Risikostrukturausgleichs (Morbi-RSA) gesetzlich festgehalten. Im Vorgriff darauf erfolgte ab 2002 die Berücksichtigung der Teilnahme an DiseaseManagement-Programmen als zusätzliches Kriterium und es wurde ein Risikopool eingerichtet. ${ }^{6}$

Die Einführung des Morbi-RSA war ursprünglich zum 1. Januar 2007 geplant, wurde aber nachträglich verzögert, um parallel den Gesundheitsfonds ${ }^{7}$ einzuführen. Beides wurde dann zum 1. Januar 2009 implementiert. Um die Ungenauigkeiten durch die im Rahmen des RSA angewandten Kriterien weiter zu reduzieren, orientiert sich der Morbi-RSA neben den Kriterien Alter, Geschlecht und Erwerbsminderungsrente an 50 bis 80 definierten und eng abgrenzbaren Krankheiten des einzelnen Versicherten. ${ }^{8}$ Dabei handelt es sich um teure chronische Erkrankungen oder Krankheiten mit schwerem Verlauf, ${ }^{9}$ deren durchschnittlichen Leistungsausgaben pro betroffenen Patienten den Durchschnitt aller Versicherten um mindestens 50 Prozent übersteigen.

\footnotetext{
${ }^{6}$ Vgl. Schulenburg, J.-M./Vieregge, D. (2003), S. 240; AOK-Bundesverband (Hrsg.) (o.J.).

${ }^{7}$ Der Gesundheitsfonds wird vom BVA als Sondervermögen verwaltet und enthält vor allem die gezahlten Beiträge und den Steuerzuschuss des Bundes (vgl. BVA (Hrsg.) 2008, S. 3).

${ }^{8}$ Vgl. BVA (Hrsg.) (2008), S. 3; § 268 Abs. 1 SGB V.

${ }^{9}$ Vgl. § 268 Abs. 1 SGB V.
} 
In der Praxis erfolgt die Zuordnung eines Patienten in eine der Gruppen im Rahmen eines stationären Aufenthaltes oder einer wiederholten ambulanten Diagnose evtl. kombiniert mit einem entsprechenden Therapienachweis (z.B. Verschreibungsdaten basierend auf der Anatomisch-Therapeutisch-Chemischen (ATC) Klassifikation mit definierten minimalen Tagesdosen (DDD, Defined Daily Doses)). Basis für die Berechnung der Zuweisung aus dem Gesundheitsfonds bilden die durchschnittlichen Pro-Kopf-Ausgaben aller GKV-Versicherten. ${ }^{10}$ Darauf aufbauend erfolgen für jeden Versicherten $\mathrm{Zu}$ - und Abschläge. ${ }^{11}$

Anhand von Alter und Geschlecht erfolgt im Morbi-RSA die Bildung von 40 Gruppen in Alterskohorten von je 5 Jahren getrennt für Männer und Frauen. Neugeborene werden separat betrachtet. ${ }^{12}$ Weiterhin gibt es sechs Erwerbsminderungsgruppen, die wiederum nach Alter und Geschlecht differenziert werden, und das Kriterium der längerfristigen Erwerbsminderungsrente. ${ }^{13}$ Die 80 festgelegten Krankheiten werden in Morbiditätsgruppen für ähnlich teure Indikationen oder unterschiedliche hierarchische Schweregrade der gleichen Diagnose aufgeteilt. Die krankheitsbedingten Zuschläge sind prospektiv ausgestaltet, das heißt sie berücksichtigen die Durchschnittskosten einer Erkrankung erst in dem Jahr, das auf die Diagnosestellung folgt und vernachlässigen die Kosten im ersten Jahr der Erkrankung.

Beispielsweise wurden Ende 2008 erstmals anhand der individuellen Diagnosen und Arzneimittelverordnungen aus dem Jahr 2006 und den individuellen Kosten des Jahres 2007 die Grundpauschale und die Zuschläge für die vorläufigen Zahlungen für das Jahr 2009 berechnet. Die Abschlagzahlungen erfolgen monatlich und werden an die Entwicklung der Versichertenzahlen der einzelnen Krankenkasse angepasst. ${ }^{14}$ Im Folgejahr, im Beispiel also 2010, erfolgt dann anhand der erforderlichen neuen Datenmeldung die endgültige rückwirkende Abrechnung mit der Krankenkasse.

Die Zuweisungen aus dem Gesundheitsfonds nach den Berechnungen des MorbiRSA machen den größten Teil der Einnahmen einer Krankenkasse aus. Hinzu kommen noch Zahlungen für sonstige Ausgaben nach § 270 SGB V. Hierunter fallen Aufwendungen für Disease Management Programme, Satzungs- und Zusatzleistungen sowie standardisierte Verwaltungsausgaben. Reichen die Zuweisungen aus dem Gesundheitsfonds nicht aus, so kann jede Kasse einen kassenindividuellen einkommensunabhängigen Zusatzbeitrag bei ihren Mitgliedern erheben, um zusätzliche Einnahmen $\mathrm{zu}$ generieren. ${ }^{15}$

Damit sind die Einnahmemöglichkeiten einer einzelnen Krankenkasse weitgehend erschöpft. Sollte darüber hinaus Finanzbedarf bestehen, ist auch eine Insolvenz möglich. ${ }^{16}$ Droht eine solche, bestehen noch Möglichkeiten zur Finanzierung einer Kasse.

\footnotetext{
${ }^{10}$ Bspw. betrug für 2009 der monatliche Grundbetrag pro Versicherten 185,6373 Euro (vgl. BVA (Hrsg.) (o.J.), S. 1).

${ }^{11}$ Vgl. BVA (Hrsg.) (2008), S. 4.

${ }^{12}$ Vgl. BVA (Hrsg.) (2008), S. 6.

${ }^{13}$ Vgl. BVA (Hrsg.) (2008), S. 6.

${ }^{14} \mathrm{Vgl}$. 39 Abs. 2 RSAV.

${ }^{15}$ Vgl. $\$ 242$ SGB V.

${ }^{16}$ Vgl. § 171b SGB V. Z.B. wurden die City BKK am 01.07.2011 (vgl. Sinß, F. (2011a), S. 682) und die BKK Heilberufe zum Jahresende 2011 durch das BVA geschlossen (vgl. Sinß, F. (2011b), S. 1626).
} 
Hierunter fallen finanzielle Hilfen des Spitzenverbandes Bund der Krankenkassen zur Ermöglichung und Erleichterung einer Vereinigung ${ }^{17}$ und freiwillige Finanzstützen innerhalb derselben Kassenart. ${ }^{18}$ Eine gefährdete Krankenkasse kann auch mit einer anderen zwangsvereinigt werden. ${ }^{19}$

\subsection{Risikoszenarien einer Krankenkasse}

Die Risiken einer gesetzlichen Krankenkasse ergeben sich primär aus den Charakteristika des Versichertenkollektivs, vor allem, weil aufgrund des Kontrahierungszwangs nach $\S 175$ Abs. 1 SGB V kaum aktive Einflussmöglichkeiten auf die Zusammensetzung der Versicherten bestehen. ${ }^{20}$ Daher werden nachfolgend potentielle Szenarien exemplarisch illustriert, welchen Risiken anhaften, die durch den MorbiRSA nicht vollständig berücksichtigt werden und die daher insbesondere kleineren Krankenkassen Probleme bereiten können.

\section{Szenario 1: Epidemie oder Pandemie ${ }^{21}$}

Dieses Szenario ist dadurch charakterisiert, dass die Gesamtheit bzw. ein großer Teil des versicherten Kollektivs gleichzeitig von einer Erkrankung betroffen ist und erhöhte Kosten verursacht. ${ }^{22}$ Das Szenario soll durch zwei Beispiele illustriert werden.

Im Jahr 2003 sorgte der Ausbruch des „Severe Acute Respiratory Syndrome“ (SARS) weltweit für Aufsehen. Die vermutlich primär per Tröpfcheninfektion übertragene Infektionskrankheit breitete sich vor allem entlang der Routen des internationalen Flugverkehrs aus. ${ }^{23}$ Für die Zeit von November 2002 bis Anfang August 2003 berichtete die WHO von 8.422 wahrscheinlichen Fällen, mit 916 Toten aus 29 Ländern. ${ }^{24}$

In Deutschland gab es im Jahr 2006 in Nordrhein-Westfalen einen Masernausbruch mit 1.749 Fällen und zwei Toten, der auch für hohe Kosten bei den Krankenkassen verantwortlich war. Die Ausbreitung konnte weitestgehend durch das Fehlen einer entsprechenden Impfung begründet werden. ${ }^{25}$

\footnotetext{
${ }^{17} \mathrm{Vgl} . \S 265$ a SGB V.

${ }^{18} \mathrm{Vgl}$. § 265b SGB V.

${ }^{19}$ Vgl. § 172 SGB V.

${ }^{20}$ Weitere Risiken sind vor allem in unternehmerischen, personellen und operativen Bereichen zu sehen und unterscheiden sich in der Regel nicht von anderen Branchen. Daher werden diese im Rahmen dieses Artikels nicht weiter betrachtet.

${ }^{21}$ Epidemie: ,,massenhaftes Auftreten einer Krankheit, v.a. einer Infektionskrankheit, in einem begrenzten Gebiet u. Zeitraum“. Roche (Hrsg.) (2006); Pandemie: ,,auf große Gebiete eines Landes oder Erdteils übergreifende Epidemie“. Roche (Hrsg.) (2006).

${ }^{22}$ Die Ausgaben einer Krankenkasse könnten aber auch durch eine ungünstige Regionalstruktur systematisch erhöht sein. Beispielsweise hatte die City BKK viele Versicherte in Ballungsräumen, die tendenziell überdurchschnittliche Kosten verursachen, die nicht vom Morbi-RSA ausgeglichen werden. Vgl. Sinß, F. (2011a). Diese Konstellation soll nicht Gegenstand der Betrachtung sein.

${ }^{23}$ Vgl. WHO (Hrsg.) (2003a), S. 17.

${ }^{24} \mathrm{Vgl}$. WHO (Hrsg.) (2003b).

${ }^{25} \mathrm{Vgl}$. Wichmann, O./Siedler, A./Sagebiel, D./Hellenbrand, W. et al. (2009).
} 


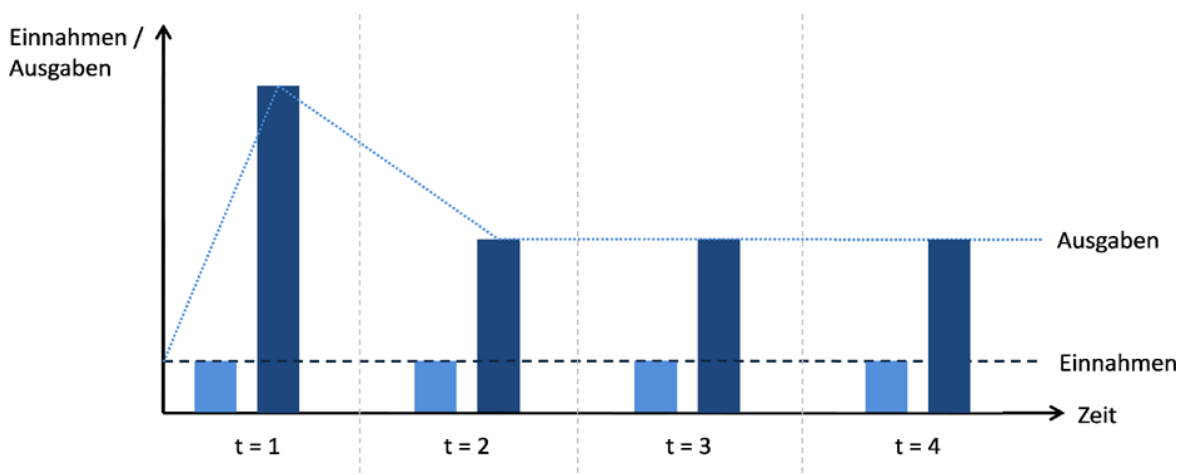

Abb. 1 Einnahmen und Ausgaben Szenario 2 im Zeitverlauf

Szenario 2: Teure chronische Krankheit, die nicht durch den Morbi-RSA abgedeckt wird

Krankenkassen erhalten für chronische Erkrankungen eines Versicherten, welche nicht von den 80 Krankheiten erfasst werden, aber dennoch dauerhaft überdurchschnittliche Kosten erzeugen, keine zusätzlichen Zuweisungen aus dem Morbi-RSA. Die Liste der berücksichtigten Erkrankungen wird regelmäßig angepasst. Die folgende Abb. 1 illustriert einen solchen Fall. Die Ausgaben übersteigen die konstanten Einnahmen ab dem Jahr der Erstdiagnose dauerhaft.

So wird z.B. seit 2011 das Aufmerksamkeitsdefizit-Hyperaktivitätssyndrom (ADHS) nicht mehr im Morbi-RSA berücksichtigt, da die durchschnittlichen Ausgaben für die Patienten nunmehr nicht das 1,5-fache des Gesamtdurchschnitts ausmachen. ${ }^{26}$ Als weitere Beispiele können seltene Erkrankungen dienen. Diese werden wegen der geforderten „Prävalenzgewichtung“ ${ }^{27}$ der berücksichtigten Krankheiten durch den Morbi-RSA weitestgehend nicht erfasst. ${ }^{28}$

Szenario 3: Teure bzw. überdurchschnittlich teure chronische Erkrankung, die vom Morbi-RSA nur teilweise ausgeglichen wird

Unter dieses Szenario fallen Patienten, deren chronische Erkrankung zwar zu den 80 im Morbi-RSA berücksichtigten Krankheiten zählt, deren Kosten für eine individuelle Krankenkasse aber durch den Morbi-RSA nur zum Teil ausgeglichen werden. Dies sind Fälle, die im Zeitverlauf regelmäßig teurer als die durchschnittlichen Krankheitskosten für die entsprechende Diagnose sind. Abbildung 2 zeigt exemplarisch einen solchen Fall: Die Einnahmen aus dem prospektiv ausgestalteten Morbi-RSA steigen zwar im Jahr nach der ersten Diagnose an, aber die Ausgaben werden dadurch langfristig nicht kompensiert.

\footnotetext{
${ }^{26}$ Vgl. Meißner, M. (2010), S. 388.

${ }^{27}$ Die berücksichtigten Krankheiten werden, neben den Kosten, auch anhand der Prävalenz bestimmt, damit diese ,für das Versorgungsgeschehen von besonderer Bedeutung sind und wesentlichen Einfluss auf die Kostenbelastung der Krankenkassen haben.“ Deutscher Bundestag (Hrsg.) (2006), S. 204.
}

${ }^{28}$ Vgl. BVA (Hrsg.) (2009a), S. 10-11. 


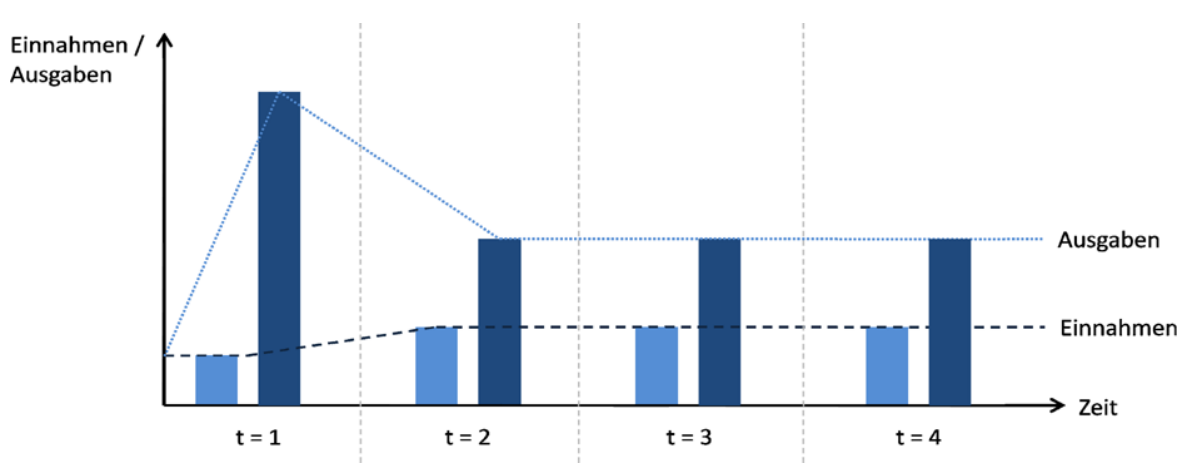

Abb. 2 Einnahmen und Ausgaben Szenario 3 im Zeitverlauf

Ein Beispiel für ein solches Risiko Liefert die Gemeinsame Betriebskrankenkasse Köln (GBK), die vor allem wegen zweier Hämophilie-Patienten als erste Kasse in Deutschland einen Zusatzbeitrag erheben musste. Innerhalb zweier Jahre verursachten die für die Behandlung notwendigen Medikamente Ausgaben von 14 Millionen Euro. $^{29}$

Die Szenarien 2 und 3 haben ähnliche Auswirkungen auf den Zahlungsstrom der Krankenkasse. Der Unterschied besteht darin, dass die anfallenden Kosten in Szenario 2 gar nicht und in Szenario 3 unvollständig durch den Morbi-RSA ausgeglichen werden. Bei beiden ist die Differenz zwischen den Einnahmen aus dem Gesundheitsfonds und den Ausgaben für den jeweiligen Versicherten langfristig negativ. Daher werden diese beiden Szenarien im weiteren Verlauf gemeinsam betrachtet.

Szenario 4: Teure (chronische) Krankheit, die insbesondere anfänglich hohe Kosten verursacht, aber langfristig vom Morbi-RSA abgedeckt wird

Dieses Szenario umfasst Patienten mit langfristig erhöhten Kosten, deren Erkrankung aber zu den 80 berücksichtigten zählt. Wie in Abb. 3 dargestellt, werden durch die prospektive Ausgestaltung des Morbi-RSA die Ausgaben des ersten Jahres nicht und die der Folgejahre vollständig ausgeglichen. Letzteres gilt, wenn die Ausgaben für den betrachteten Patienten den Durchschnitt der entsprechenden Gruppe nicht übertreffen.

Hier wäre z.B. an eine Organtransplantation ${ }^{30} \mathrm{zu}$ denken. Es entstehen entsprechende hohe Kosten durch die anfängliche Operation und im Vergleich geringere Kosten für die Nachsorge.

Szenario 5: Teure akute Krankheit, die aufgrund der prospektiven Ausgestaltung des Morbi-RSA nicht abgedeckt wird

Einmalige akute Erkrankungen sollen durch den Morbi-RSA nicht ausgeglichen wer-

\footnotetext{
${ }^{29}$ Vgl. Berger, P. / Wiedemann, G. M. (2009). Der Zuschlag für Hämophilie betrug z.B. 2009 ca. 5000 Euro pro Monat (vgl. BVA (Hrsg.) (o.J.), S. 4).

30 „Status nach Organtransplantation (inkl. Komplikationen)“ ist eine der berücksichtigten Krankheiten, vgl. BVA (Hrsg.) (2009b), S. 3.
} 


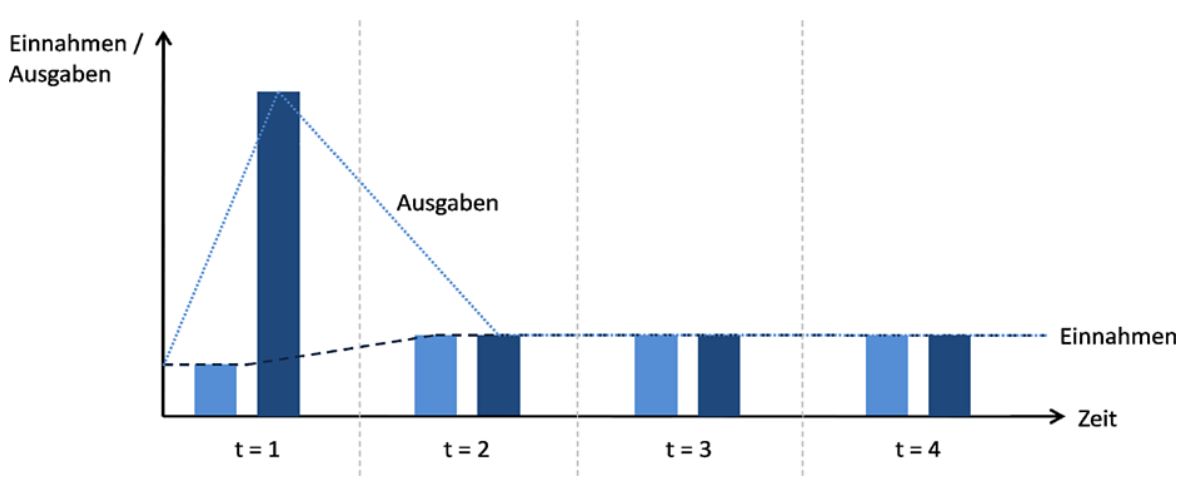

Abb. 3 Einnahmen und Ausgaben Szenario 4 im Zeitverlauf

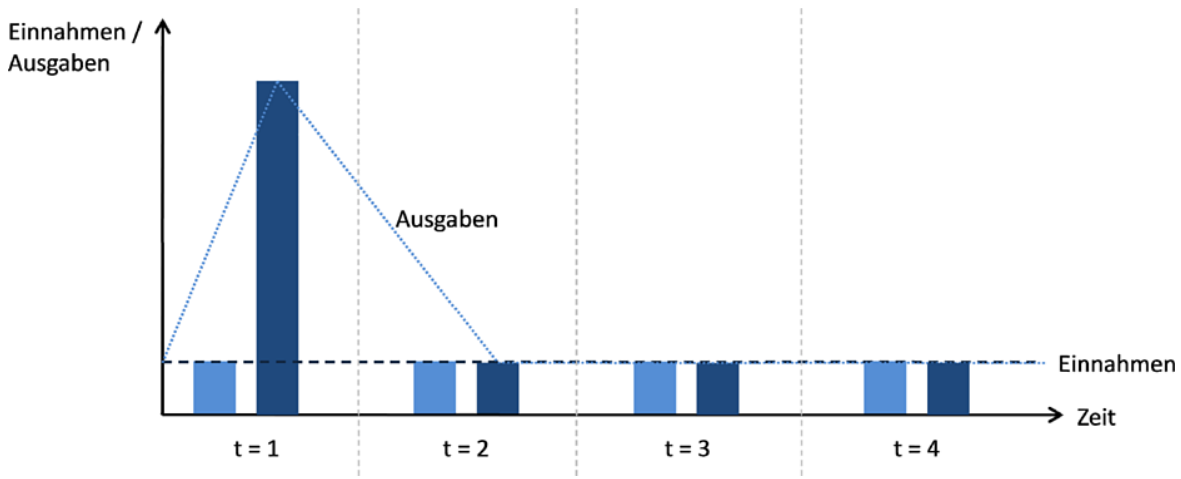

Abb. 4 Einnahmen und Ausgaben Szenario 5 im Zeitverlauf

den. ${ }^{31}$ Dies gilt auch, wenn die Kosten für den Patienten über einen kurzen Zeitraum sehr hoch sind. Als Beispiele können Infektionen, eine Blinddarmentzündung ${ }^{32}$ und andere einmalige chirurgische Eingriffe genannt werden, die im Anschluss zu keinen weiteren Kosten führen. Dies findet sich auch in Abb. 4 wieder: Die Erkrankung des Versicherten wirkt sich auf die Einnahmen der Krankenkasse nicht und auf die Ausgaben nur in einer Periode aus.

Auch die Szenarien 3 und 4 können gemeinsam betrachtet werden. Der MorbiRSA ist prospektiv ausgestaltet und soll von daher nicht die initialen Kosten einer Krankheit abdecken. In beiden Szenarien übersteigen die Ausgaben die Einnahmen daher nur in der Periode der Erstdiagnose. In der Folge werden evtl. anfallende höhere Kosten durch den Morbi-RSA abgedeckt (Szenario 4) oder fallen gar nicht erst an (Szenario 5).

Die fünf entwickelten Szenarien zeigen, dass es trotz Morbi-RSA krankheitsbedingte Kosten bei Krankenkassen gibt, welche nicht ausgeglichen werden. Entspre-

${ }^{31}$ BVA (Hrsg.) (2008), S. 8.
${ }^{32}$ BVA (Hrsg.) (2008), S. 9. 
chend entstehen den Versicherungen hieraus finanzielle Risiken. Zusammenfassend ergeben sich für die weiteren Untersuchungen drei Konstellationen basierend auf dem Szenario 1, den jeweils gemeinsam zu betrachtenden Szenarien 2 und 3 sowie 4 und 5 als Risiken für eine mögliche Insolvenz einer Krankenkasse.

Im Folgenden werden Kriterien vorgestellt, anhand derer die Versicherbarkeit von Risiken analysiert werden kann. Im Anschluss daran werden die aus den Szenarien folgenden Risiken entsprechend dieser Versicherbarkeitskriterien analysiert.

\section{Kriterien der Versicherbarkeit}

Im Allgemeinen kann ein Risiko als versicherbar gelten, sobald ein Versicherer dieses Risiko analysiert, einen Preis kalkuliert und, falls der Versicherungskunde die angebotene Prämie annimmt, das Risiko zeichnet. ${ }^{33}$ Dieses Vorgehen zur Bestimmung der Grenzen der Versicherbarkeit wird als empirisch-induktiver Ansatz bezeichnet. Allerdings ist dieser in der Regel vor allem bei bekannten sowie kalkulierbaren, aber weniger für neuartige Risiken anwendbar. ${ }^{34}$ Alternativ dazu entwickelt Karten einen logisch-deduktiven Ansatz, anhand der im Folgenden kurz erläuterten Kriterien der Versicherbarkeit von Risiken: ${ }^{35}$

- Zufälligkeit des Schadenereignisses

Dieses Kriterium bezieht sich darauf, dass beim Versicherer und beim Versicherten Unsicherheit über den tatsächlichen Schadeneintritt und weiterhin über Zeitpunkt und/oder Ausmaß des Schadens herrschen müssen. ${ }^{36}$ Der Versicherer hat dabei zusätzlich Verhaltensänderungen durch den Versicherten („,moral hazard“) zu berücksichtigen. ${ }^{37}$

- Eindeutigkeit der Versicherungsleistung

Darunter ist das Festlegen eines Ereignisses und des in diesem Zusammenhang zu leistenden Geldbetrages vor Vertragsschluss zu verstehen. ${ }^{38}$

- Schätzbarkeit der Schadenwahrscheinlichkeit

Dabei geht es darum Aussagen über die Eintrittswahrscheinlichkeit und das Ausmaß eines Schadens zu treffen, um eine auskömmliche Prämie zu berechnen.

- Unabhängigkeit der Risiken

Die einzelnen Schäden sollten demnach nicht positiv korreliert sein, womit weder ein Kumulrisiko noch eine Eintrittsabfolge (Ansteckungsrisiko) vorliegen. Insgesamt sind viele Risiken jedoch miteinander korreliert. In dem Fall geht es darum,

\footnotetext{
${ }^{33}$ Vgl. Benzin, A. (2005), S. 237.

${ }^{34}$ Vgl. Höller, J. (1997), S. 231-232.

${ }^{35}$ Vgl. Karten, W. (1972), S. 287 ff. Neben diesen Kriterien nach Karten finden sich weitere Kriterienkataloge. So z.B. Berliner, B. (1982) zit. nach Berliner, B. (1988), S. 953 und Hausmann, P. (1998), S. 7. Zu einer kritischen Diskussion verschiedener Kriterien vgl. Höller, J. (1997), S. $230 f f$.

${ }^{36}$ Vgl. Karten, W. (1972), S. 287.

${ }^{37}$ Vgl. Schanz, K.-U./Fehr, K./Armitage, T. (2010), S. 34; Karten, W. (1972), S. 288.

${ }^{38}$ Vgl. Karten, W. (1972), S. 288.
} 
dass die Höhe der Korrelation begrenzt ist ${ }^{39}$ und/oder ein Ausgleich in der Zeit möglich ist. ${ }^{40}$

- Begrenzung des größtmöglichen Schadens

Hierbei handelt es sich um eine Abschätzung der Schadenhöhe im schlimmsten anzunehmenden Fall. Selbst dieser Schaden sollte noch durch die Kapazitäten des Versicherers abzudecken sein. ${ }^{41}$

Nicht erst seit den Anschlägen in New York vom 11. September 2001 werden die Grenzen und die Kriterien der Versicherbarkeit stark diskutiert. ${ }^{42}$ Im Zeitverlauf finden sich verschiedene Kriterienkataloge, die meist nur geringe inhaltliche Abweichungen aufweisen. ${ }^{43}$ Bei der Entscheidung ob ein Risiko letztlich versichert wird, hat u.a. auch das vorhandene Kollektiv des fraglichen Versicherers mit seiner kumulierten Schadenverteilung einen großen Einfluss. ${ }^{44}$ Die Einschätzung der Versicherbarkeit verändert sich auch mit zunehmender Erfahrung, Information und Kapazität. ${ }^{45}$ Trotz der Diskussion um die (generelle) Anwendbarkeit von Kriterienkatalogen und auch deren jeweiligen speziellen Ausprägungen, werden im weiteren Verlauf die beschriebenen Kriterien nach Karten verwendet. Diese reichen für eine erste Annäherung an die Frage, inwieweit Versicherungslösungen für Risiken in der gesetzlichen Krankenversicherung adäquat erscheinen und die Analyse der beschriebenen Szenarien zunächst aus.

\section{Analyse der Risikoszenarien hinsichtlich ihrer Versicherbarkeit}

Szenario 1: Epidemie oder Pandemie In diesem Szenario sind die Kriterien „Zufälligkeit des Schadenereignisses“ und „Eindeutigkeit der Versicherungsleistung“ erfüllbar. Probleme bereitet hingegen die „Schätzbarkeit der Schadenwahrscheinlichkeit“. In der Vergangenheit sind zum Glück nur sehr wenige wirkliche Epidemien oder Pandemien in Europa aufgetreten. Die letzten Beispiele für eine gefürchtete weltweite Ausbreitung liefern SARS im Jahr 2002/2003 und die sogenannte Schweinegrippe im Jahr 2009. Hieran zeigt sich auch, dass insbesondere durch die gestiegene internationale Mobilität eine Schätzung der Ausbreitung sehr schwierig ist.

Probleme treten weiterhin bei den Kriterien der „Unabhängigkeit der Risiken“ und der „Begrenzung des größtmöglichen Schadens“ auf. Epidemien/Pandemien sind gerade durch das Auftreten von Kumulrisiken und der damit verbundenen Verletzung des Unabhängigkeitskriteriums gekennzeichnet. Auch der mögliche Schaden kann je nach Krankheitsbild sehr hoch werden, da immer eine Vielzahl von Versicherten

\footnotetext{
${ }^{39}$ Vgl. Nguyen, T. (2008), S. 7.

${ }^{40}$ Vgl. Karten, W. (1972), S. 292.

${ }^{41}$ Vgl. Karten, W. (1972), S. 292.

${ }^{42}$ Vgl. Seitz, M. (2002), S. 35.

${ }^{43}$ Vgl. hierzu z.B. Höller, J. (1997), S. 240.

${ }^{44}$ Vgl. Karten, W. (1972), S. 285.

${ }^{45}$ Vgl. Goßner, A. (2002), S. 6.
} 
betroffen ist. An dieser Stelle ist allerdings die Schadenbegrenzung seitens des Rückversicherers z.B. durch die vorherige Festlegung einer Versicherungssumme möglich. Hierdurch und z.B. durch Selbstbeteiligungen würde auch der theoretisch mögliche Anreiz zum Moral Hazard durch die risikoabgebende Krankenkasse begrenzt.

Allerdings unterliegen alle Beteiligten bei Pandemien auch besonderen gesetzlichen Regelungen. Ein nationaler Pandemieplan für Deutschland wird auf der Ebene der einzelnen Bundesländer umgesetzt. ${ }^{46}$ Daher scheinen die Risiken der einzelnen Krankenkasse begrenzt, da in diesem Fall staatliche Eingriffe und finanzielle Hilfe notwendig werden.

Szenarien 2 und 3: Teure chronische Krankheit, die nicht oder unvollständig vom Morbi-RSA ausgeglichen wird Die Szenarien 2 und 3 wurden zusammengefasst, da jeweils Erkrankungen zugrunde liegen, welche für die versichernden Krankenkassen langfristig höhere Ausgaben als Einnahmen generieren. In einem Fall ist die Krankheit nicht in den 80 berücksichtigten enthalten, im anderen Fall reichen die Zuweisungen nicht aus, da der individuelle Patient überdurchschnittliche Kosten verursacht.

Vor dem Hintergrund der Informationen, die dem Erstversicherer, also im betrachteten Fall der Krankenkasse, vorliegen, sind die Kriterien der „Zufälligkeit des Schadenereignisses“ und der „Unabhängigkeit der Risiken“ weitgehend erfüllt.

Die verbleibenden Kriterien „Eindeutigkeit“, „Schätzbarkeit“ und „Begrenzung des größtmöglichen Schadens“ sind schwieriger zu beurteilen. Hauptproblem hierbei ist, dass die Folgekosten eines chronisch Erkrankten sowohl in der Höhe als auch in der Zeit schwer schätzbar sind. So können chronisch Kranke (wie z.B. Diabetiker) durchaus noch mehrere Jahrzehnte mit der Krankheit leben, in denen aber immer Kosten der Krankheit z.B. durch Medikamente entstehen. Sollten diese laufenden Zahlungen durch eine Rückversicherung gedeckt sein, besteht für den Erstversicherer ein verringerter Anreiz zur Versorgungsverbesserung. Ein weiteres Feld der Unsicherheit bieten Co-Morbiditäten, deren Kosten schwer von der evtl. ursächlichen Grunderkrankung trennbar sind und somit Folgekosten der Originalerkrankung darstellen könnten.

Eine Möglichkeit besteht darin, zum Zeitpunkt der sicheren Diagnose der Krankheit eine einmalige barwertige oder vorher vertraglich festgelegte fixe Zahlung an den Erstversicherer zu leisten. Natürlich entstehen auch daraus weitere praktische Probleme, wie die Festlegung des Zeitpunkts der sicheren Diagnose (Eindeutigkeit), der Berechnung des Barwertes (Schätzbarkeit) oder der Festlegung einer Versicherungssumme (Begrenzung des größtmöglichen Schadens) ohne die Möglichkeit der Bereicherung. Theoretisch ist das grundlegende Problem der Rückversicherbarkeit auf diese Weise lösbar. Allerdings besteht noch erheblicher Forschungsbedarf bezüglich der Durchführung der damit verbundenen Berechnungen und der Verhinderung von (zumindest theoretisch möglichem) Moral Hazard durch die Krankenkasse. ${ }^{47}$

\footnotetext{
${ }^{46}$ Vgl. Thiele, O./Thiele, M. (o.J.).

${ }^{47}$ Seit der Einführung des Morbi-RSA gibt es ähnliche Diskussionen unter dem Stichwort des ,upcoding“. Vgl. hierzu z.B. Gaßner, M./Arndt, V./Fischer, L. et al. (2010).
} 
Szenarien 4 und 5: Teure Krankheit (chronisch oder akut), die nur in der ersten Periode nicht von den Zahlungen des Morbi-RSA gedeckt wird Diese Szenarien werden gemeinsam betrachtet, da bei beiden nur im Jahr der Diagnose eine negative Differenz zwischen Einnahmen und Ausgaben zu verzeichnen ist. Der Unterschied besteht darin, dass in einem Fall die langfristigen Kosten der Patienten nicht erhöht sind, z.B. weil die Gesundheit komplett wiederhergestellt ist. Im anderen Fall werden die langfristigen Kosten vom Morbi-RSA erfasst und vollständig ausgeglichen.

In diesen Fällen handelt es sich um einen „klassischen“ Versicherungsfall. Gerade akute Erkrankungen (z.B. durch einen Unfall verursachte) erfüllen explizit die Eigenschaften der Versicherbarkeit. Sie treten zufällig und unabhängig voneinander auf, sind sowohl als Ereignis als auch in der Schadenhöhe weitestgehend eindeutig sowie, bedingt durch den Leistungsumfang der Gesetzlichen Krankenkassen, einigermaßen begrenzt. Probleme können auf der Ebene der Schätzbarkeit bestehen, da evtl. zu einem späteren Zeitpunkt Folgeerkrankungen auftreten, bei denen Uneinigkeit über den Zusammenhang mit der Ursprungserkrankung herrscht. Insgesamt sind diese Risiken allerdings von geringer Relevanz, da sie das tägliche Geschäft einer Gesetzlichen Krankenkasse abbilden.

\section{Diskussion und weiterer Forschungsbedarf}

Insgesamt betrachtet existieren Risiken auf Seiten der Krankenkassen, welche die Voraussetzung der Rückversicherbarkeit erfüllen. Insbesondere kleinere Krankenkassen, die häufig auch ein regional konzentriertes Versichertenkollektiv haben, können von einer Rückversicherungslösung profitieren, da somit der Ausgleich in einem größeren Kollektiv realisierbar wird, ohne z.B. mit einer anderen Kasse zu fusionieren. Größere Krankenkassen haben dahingegen bessere Möglichkeiten den Risikoausgleich im eigenen Kollektiv zu erreichen, weshalb Rückversicherungslösungen für diese weniger interessant sind. Beachtung sollte allerdings auch den negativen Anreizen gezollt werden, die mit einer (Rück-)Versicherungslösung einhergehen. Dies gilt auch vor dem Hintergrund, dass der Rückversicherer keine unternehmerischen Risiken des Zedenten tragen sollte.

Die vorliegende Analyse ist rein deskriptiv und szenarienbasiert. Weiterer Forschungsbedarf besteht vor allem in der Modellierung der Risiken und der Kalkulation nach versicherungsmathematischen Prinzipien. Auch die rechtliche Organisation der Rückversicherungslösungen ist zu prüfen. Darüber hinaus bieten neuere Formen der medizinischen Versorgung, wie z.B. Ärztenetze, Medizinische Versorgungszentren, Integrierte Versorgung etc. weiteres Versicherungs- und damit gegebenenfalls auch Rückversicherungspotential. In diesen Versorgungsformen wird derzeit zum Teil mit Kopfpauschalen vergütet, womit ein großer Teil des Risikos bei den Betreibern verbleibt. ${ }^{48}$ Durch die Einbindung und Nutzung des Know-Hows erfahrener und unabhängiger Rückversicherer könnte Planungssicherheit geschaffen und die Effizienz der Versorgung gesteigert werden. Hier stellt sich zusätzlich die Frage, wer der Risikoträger ist, der als Erstversicherer fungiert und so Zugang zum Rückversicherungsmarkt hat.

${ }^{48}$ Vgl. Schlingensiepen, I. (2010). 


\section{Literatur}

AOK-Bundesverband (Hrsg.): Die Geschichte des Risikostrukturausgleichs. URL: http://www.aok-bv.de/ politik/finanzierung/morbi-rsa/index_00994.html [Stand 20.12.2011]. (o.J.)

Benzin, A.: Versicherungstechnische Bewertung unterschiedlicher Deckungskonzepte für Terrorismusrisiken. In: Karlsruher Reihe II - Risikoforschung und Versicherungsmanagement, VVW Karlsruhe, Bd. 4 (2005)

Berger, P., Wiedemann, G.M.: 14 Millionen Kosten für zwei Patienten. In: Kölner Stadtanzeiger (2009). URL: http://www.ksta.de/html/artikel/1246883788785.shtml [Stand 30.12.11]

Berliner, B.: Limits of Insurability of Risks, Englewood-Cliffs (1982)

Berliner, B.: Versicherbarkeit. In: Farny, D., Helten, E., Koch, P., Schmidt, R. (Hrsg.) Handwörterbuch der Versicherung HdV, VVW Karlsruhe (1988)

BKK-Landesverband Nordwest (Hrsg.): Anlage „Ausgleichsordnung“: Satzung des BKKLandesverbandes Nordwest, Stand: 20. Oktober 2011 (2011). URL: http://www.bkk-nordwest.de/ wir_ueber_uns/satzung/download/satzung_2011-10-20.pdf [Stand 13.12.2011]

BVA (Hrsg.): So funktioniert der neue Risikostrukturausgleich im Gesundheitsfonds, Bundesversicherungsamt 2008 (2008). URL: http://www.bundesversicherungsamt.de/cln_100/nn_1046668/DE/ Risikostrukturausgleich/Wie_funktioniert_Morbi_RSA,templateId=raw,property=publicationFile. pdf/Wie_funktioniert_Morbi_RSA.pdf [Stand 20.12.2011]

BVA (Hrsg.): Erläuterungen zur Festlegung der im morbiditätsorientierten Risikostrukturausgleich für das Ausgleichsjahr 2010 zu berücksichtigenden Krankheiten durch das Bundesversicherungsamt (2009a). URL: http://www.bundesversicherungsamt.de/cln_115/nn_1440668/DE/ Risikostrukturausgleich/Festlegungen/Festlegung_Krankheiten_30092009,templateId=raw,property= publicationFile.zip/Festlegung_Krankheiten_30092009.zip [Stand 13.12.2011]

BVA (Hrsg.): Festlegung der im Jahresausgleich 2010 zu berücksichtigenden Krankheiten nach § 31 Abs. 4 S. 1 RSAV (2009b). URL: http://www.bundesversicherungsamt.de/cln_115/nn_1440668/DE/ Risikostrukturausgleich/Festlegungen/Festlegung_Krankheiten_30092009,templateId=raw,property =publicationFile.zip/Festlegung_Krankheiten_30092009.zip [Stand 13.12.2011]

BVA (Hrsg.): Vorabinformation - Zuweisungen 2009 aus dem Gesundheitsfonds, (o.J.). URL: http:// www.bundesversicherungsamt.de/cln_108/nn_1046648/DE/Gesundheitsfonds/Zuweisungen/ Vorabinfo_Zuweisungen,templateId=raw,property=publicationFile.pdf/Vorabinfo_Zuweisungen.pdf [Stand: 16.12.2011]

Deutscher Bundestag (Hrsg.): Entwurf eines Gesetzes zur Stärkung des Wettbewerbs in der gesetzlichen Krankenversicherung (GKV-Wettbewerbsstärkungsgesetz - GKV-WSG), Deutscher Bundestag, Drucksache 16/3100 (2006). URL: http://dipbt.bundestag.de/dip21/btd/16/031/1603100.pdf [Stand: 29.12.2011]

Gaßner, M., Arndt, V., Fischer, L., et al.: Sind die Diagnosezahlen nach Einführung des morbiditätsorientierten Risikostrukturausgleichs angestiegen? Gesundheits- und Sozialpolitik - Z. gesamte Gesundheitswesen 6, 11-19 (2010)

Goßner, A.: Gibt es neue unversicherbare Risiken. In: Hamburger Gesellschaft zur Förderung des Versicherungswesens mbH (Hrsg.) Der Umgang mit den Risiken im Grenzbereich der Versicherbarkeit - Dokumentation über ein Symposium am 18.-20. Oktober 2001 im Schloß Marbach, Öhningen, VVW Karlsruhe, S. 5-10 (2002)

Hausmann, P.: In: Schweizerische Rückversicherungs-Gesellschaft (Hrsg.) Überschwemmungen: Ein versicherbares Risiko? (1998). URL: http://media.swissre.com/documents/floods_insurable_risk_de. pdf [Stand 20.12.2011]

Höller, J.: Versicherungstechnologie: Ein Beitrag zur Diskussion theoretischer Grundlagen. In: Veröffentlichungen des Seminars für Versicherungslehre der Universität Frankfurt am Main, VVW Karlsruhe, Bd. 9 (1997).

Karten, W.: Zum Problem der Versicherbarkeit und zur Risikopolitik des Versicherungsunternehmens betriebswirtschaftliche Aspekte. Z. gesamte Versicherungswissenschaft 61, 279-299 (1972)

Meißner, M.: Weniger Geld für die Behandlung von Kindern mit ADHS ab 2011. Dtsch. Ärztebl. 9, 388 (2010)

Nguyen, T.: Versicherbarkeit von Katastrophenrisiken und staatliche Risikoübernahme. Preprint Series: 2008-06 (2008). URL: http://www.uni-ulm.de/fileadmin/website_uni_ulm/mawi/forschung/ PreprintServer/2008/Versicherbarkeit_Nguyen.pdf [Stand 20.12.2011]

Roche (Hrsg.): Roche Lexikon Medizin, 5. Aufl. (2006). URL: http://www.roche.de/lexikon/index.htm? loc $=$ www.roche.de\&content=/lexikon/suche.html [Stand 20.12.2011] 
RSAV: Risikostruktur-Ausgleichsverordnung vom 3. Januar 1994 (BGBl. I S. 55), zuletzt geändert durch Artikel 14 des Gesetzes vom 22. Dezember 2010 (BGB1. I S. 2309)

Schanz, K.-U., Fehr, K., Armitage, T.: In: Schweizerische Rückversicherungs-Gesellschaft (Hrsg.) Wegweisende Einführung in die Rückversicherung (2010). URL: http://media.swissre.com/documents/ The_Essential_Guide_To_Reinsurance_DE1.pdf [Stand 20.12.2011]

Schlingensiepen, I.: Rückversicherung im Gesundheitswesen: Teure Therapien abfedern. In: Financial Times Deutschland (2010). URL: http://www.ftd.de/unternehmen/versicherungen/: rueckversicherungen-im-gesundheitswesen-teure-therapien-abfedern/50169208.html?page=2 [Stand 20.12.2011]

Schulenburg, J.-M., Vieregge, D.: Rückversicherung und Risikostrukturausgleich als Instrumente der Risikoallokation in der gesetzlichen Krankenversicherung. Zeitschrift für die Gesamte Versicherungswissenschaft 2, 231-248 (2003)

Seitz, M.: Die Möglichkeiten des traditionellen Rückversicherungsmarktes im Umgang mit Grenzrisiken. In: Hamburger Gesellschaft zur Förderung des Versicherungswesens mbH (Hrsg.) Der Umgang mit den Risiken im Grenzbereich der Versicherbarkeit - Dokumentation über ein Symposium am 18.-20. Oktober 2001 im Schloß Marbach, Öhningen, VVW-Verlag Karlsruhe, S. 35-43 (2002)

SGB V: Fünftes Buch Sozialgesetzbuch - Gesetzliche Krankenversicherung - (Artikel 1 des Gesetzes vom 20. Dezember 1988, BGB1. I S. 2477), zuletzt geändert durch Artikel 3 des Gesetzes vom 28. Juli 2011 (BGB1. I S. 1622)

Sinß, F.: City BKK wird geschlossen. Versicherungswirtschaft 66(10), 682 (2011a)

Sinß, F.: BKK Heilberufe schließt zum Jahresende. Versicherungswirtschaft 66(22), 1626 (2011b)

Strassl, W.: Integrierten Modellen der Krankenversicherung gehört die Zukunft. Z. Versicher.wes. 21, 747748 (2010)

Thiele, O., Thiele, M.: Schweinegrippe A/H1N1 - Pandemie Pläne für Deutschland. (o.J.) URL: http:// www.schweinegrippe-h1n1.seuchen-info.de/informationen-zu-schweinegrippe-h1n1-virus/pandemieplan.html [Stand 20.12.2011]

Weber, A.: Rückversicherung - Modell für die GKV? Arb. Sozialpolit. 11, 322-326 (1989)

Weber, A.: Rückversicherung für die GKV - Modell einer Lösung zum Problem unterschiedlicher Risikostrukturen. Arb. Sozialpolit. 7-8, 10-14 (1991)

WHO (Hrsg.): World Health Organization, Department of communicable disease surveillance and response, Consensus document on the epidemiology of severe acute respiratory syndrome (SARS) (2003a). URL: http://www.who.int/csr/sars/en/WHOconsensus.pdf [Stand 13.12.2011]

WHO (Hrsg.): World Health Organization, Summary table of SARS cases by country, 1 November 2002 7 August 2003 (2003b). URL: http://www.who.int/csr/sars/country/en/country2003_08_15.pdf [Stand 13.12.2011]

Wichmann, O., Siedler, A., Sagebiel, D., Hellenbrand, W., et al.: Further efforts needed to achieve measles elimination in Germany: results of an outbreak investigation. Bull. World Health Organ. 87(2), 108115 (2009) 